\title{
Linear Regression Model on Multiresolution Analysis for Texture Classification
}

\author{
A.Subha \\ M.E Applied Electronics Student \\ Anna University Tirunelveli \\ Tirunelveli
}

\author{
S.Lenty Stuwart \\ Lecturer, ECE Department \\ Anna University Tirunelveli \\ Tirunelveli
}

\begin{abstract}
Texture is a surface property which is used to identify and recognize the object. Texture analysis is important in many applications of computer image analysis for classification and segmentation of images based on local spatial patterns of intensity or color. In texture classification the goal is to assign an unknown sample image to one set of known texture classes. The proposed method is texture analysis and classification with linear regression model based on directional lifting based wavelet transform. In this method, texture classification is performed by analyzing the spatial correlation between some sample texture images belonging to the same kind of texture at different frequency regions, obtained by 2-D wavelet transform. The linear regression model is employed to analyze this correlation and extract texture features that characterize the samples. Therefore, this method not only considers the frequency regions but also the correlation between the frequency regions. So the classification rate is improved.
\end{abstract}

\section{Keywords}

Texture analysis,Texture Classification, Linear Regression Model, Multiresolution Analysis.

\section{INTRODUCTION}

Texture is the distribution of crystallographic orientations of a sample. A sample in which these orientations are fully random is said to have no texture. If the crystallographic orientations are not random, but have some preferred orientation, then the sample has a weak, strong, or moderate texture. Texture is seen in almost all engineered materials, and it can have a great influence on material properties. Also geologic rocks show texture due to their thermo-mechanic history due to formation processes. One extreme case is a complete lack of texture: a solid with perfectly random crystallite orientation, which will have isotropic properties at length scales sufficiently larger than the size of the crystallites. The opposite extreme is a perfect single crystal, which has anisotropic properties by geometric necessity. This paper is organized as follows. The brief review about 2-D wavelet transform and an application of the correlation between different frequency regions to texture analysis are described in Section II. Section III describes the texture classification algorithm with respect to the correlation. In Section IV, some experimental results are presented. Finally, the conclusions are summarized in Section 6.

\section{DIRECTIONAL LIFTING BASED WAVELET TRANSFORM}

In wavelet lifting structure, shown in figure (1), was proposed as an alternative method to implement the wavelet transform more efficiently in the sense of minimizing the computational complexity and required memory. As shown in the figure (3), at first the signal is split into the even, $x e$ and odd, $x o$, samples; $P$ is the Prediction filter that tries to predict the odd samples from the corresponding even samples and $U$ is the Update filter that smoothes the signal $x e$. For 2-D signals, this structure is applied to the rows and columns separately. At the vertical stage all the rows are divided into the even and odd rows and the odd rows are predicted from the even rows and the even rows are updated from the predicted values of the odd rows. After that the same procedure is done for the columns. This kind of 2-D structure is very efficient in representing the horizontal and vertical 
edges. But as mentioned before, if the edges are not vertical or horizontal we will get a better description by aligning the direction of the wavelet to the direction of the edges. Combining the idea of applying the wavelet in the direction of the edges with the idea of lifting-based implementation, a simple structure for directional lifting- based wavelet transform can be devised. In the following, a brief description of the algorithm is presented.

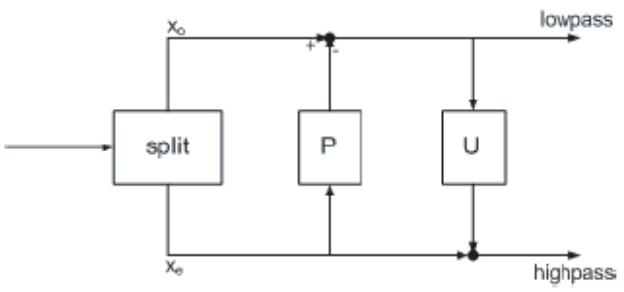

Fig.1.Block Diagram of Lifting Structure

Split: Divide the original data into two disjoint subsets.For example, we will split the original data set into even indexed points and the odd indexed points.

$\left(\right.$ even $_{\mathrm{j}-1}, \operatorname{odd}_{\mathrm{j}-1)}=\operatorname{split}\left(\mathrm{s}_{\mathrm{j}}\right)$

Predict: Generate the wavelet coefficients $d_{j-1}$ as the error in predicting odd indexed points from even indexed points using prediction operator P:

$\mathrm{d}_{\mathrm{j}-1}=\operatorname{odd}_{\mathrm{j}-1}$-even $_{\mathrm{j}-1}$

Update: Combine even indexed points and wavelet coefficients to obtain scaling coefficients $\mathrm{S}_{\mathrm{j}-1}$ that represent a coarse approximation to the original signal $\mathrm{x}[\mathrm{n}]$. This is accomplished by applying an update operator $U$ to the wavelet coefficients and adding to even indexed points:

$\mathrm{s}_{\mathrm{j}-1}=$ even $_{\mathrm{j}-1}+\mathrm{d}_{\mathrm{j}-1} / 2$

\section{ANALYZING TEXTURE WITH LINEAR REGRESSION MODEL}

\section{A. Two-Dimensional Wavelet Packet Transform}

The wavelet transform provides a precise and unifying framework for the analysis and characterization of a signal at different scales . It is described as a multiresolution analysis tool for the finite energy function $\mathrm{f}(\mathrm{x}) \in \mathrm{L} 2$. Adversely, the wavelet packet transform decomposes a signal in all low and high frequency regions.An image is divided into four subimages by using directional lifting based wavelet transform. These four subimages characterize the frequency information of the image in the LL, LH, HL, and $\mathrm{HH}$ frequency regions, respectively. The 2-D wavelet packet transform decomposes all frequency regions to achieve a full decomposition, as shown in Fig. 2. The 2-D wavelet packet transform describes the properties of the image in all regions. Most of the research in the multiresolution analysis based on the wavelet domain focuses on directly extracting the energy values from the subimages and uses them to characterize the texture image. The energy distribution of a subimage can be calculated by one of the three commonly used functions: the magnitude, the squaring, and the rectified sigmoid.

\begin{tabular}{|c|c|c|c|c|c|c|c|}
\hline $\mathrm{LL}_{3}$ & $\mathrm{HL}_{3}$ & $\mathbf{L L}_{3}$ & $\mathrm{HI}_{3}$ & $\mathrm{LL}_{3}$ & $\mathrm{HIL}_{3}$ & $\mathrm{LL}_{3}$ & $\mathrm{HIL}_{3}$ \\
\hline $\mathbf{L H}_{3}$ & $\mathbf{H H}_{3}$ & $\mathrm{LH}_{3}$ & $\mathrm{HH}_{3}$ & $\mathrm{LH}_{3}$ & $\mathbf{H H}_{3}$ & $\mathrm{LH}_{3}$ & $\mathbf{H H}_{3}$ \\
\hline $\mathrm{LL}_{3}$ & $\mathbf{H L}_{3}$ & $\mathrm{LL}_{3}$ & $\mathrm{HIL}_{3}$ & $\mathbf{L L}_{3}$ & $\mathrm{HL}_{3}$ & $\mathrm{LL}_{3}$ & $\mathrm{HIL}_{3}$ \\
\hline $\mathrm{LH}_{3}$ & $\mathrm{HH}_{3}$ & $\mathbf{L H}_{3}$ & $\mathrm{HH}_{3}$ & $\mathrm{LH}_{3}$ & $\mathbf{H H}_{3}$ & $\mathrm{LH}_{3}$ & $\mathrm{HH}_{3}$ \\
\hline $\mathrm{LL}_{3}$ & $\mathrm{HL}_{3}$ & $\mathrm{LL}_{3}$ & $\mathrm{HIL}_{3}$ & $\mathbf{L L}_{3}$ & $\mathrm{HL}_{3}$ & $\mathrm{LL}_{3}$ & $\mathrm{HIL}_{3}$ \\
\hline $\mathrm{LH}_{3}$ & $\mathrm{HH}_{3}$ & $\mathrm{LH}_{3}$ & $\mathrm{HFH}_{3}$ & $\mathrm{LH}_{3}$ & $\mathrm{HOH}_{3}$ & $\mathrm{LH}_{3}$ & $\mathrm{HH}_{3}$ \\
\hline $\mathrm{LL}_{3}$ & $\mathrm{HL}_{3}$ & $\mathbf{L L}_{3}$ & $\mathrm{HIL}_{3}$ & $\mathbf{L L}_{3}$ & $\mathrm{HL}_{3}$ & $\mathrm{LL}_{3}$ & $\mathrm{HL}_{3}$ \\
\hline $\mathbf{L H}_{3}$ & $\mathrm{HH}_{3}$ & $\mathrm{LH}_{3}$ & $\mathrm{LH}_{3}$ & $\mathrm{HH}_{3}$ & $\mathrm{HH}_{3}$ & $\mathbf{L H}_{3}$ & $\mathrm{HHH}_{3}$ \\
\hline
\end{tabular}

Fig. 2. Three level 2-D wavelet packet decomposition of $128 \times 128$ image

The mean and the standard deviation of the magnitude of the subimage coefficients also can be calculated as texture feature. In this paper, the mean of the magnitude of the 
subimage coefficients is used as its energy. That is, if the subimage is $x(m, n)$, with $1 \leq \mathrm{m} \leq \mathrm{M}$ and $1 \leq \mathrm{n} \leq \mathrm{N}$, its energy can be represented as

$$
\mathrm{e}=1 / \mathrm{MN} \sum_{j=1}^{N}|x(i, j)|
$$

where is the pixel value of the subimage.

\section{B. Analysis of Correlation Between Frequency Channels}

In this paper, the correlation does not refer to the linear correlation between different frequency regions of a texture image after decomposition by 2-D wavelet transform, but instead indicates the spatial correlation between some sample texture images, belonging to the same kind of texture, at different frequency regions obtained by 2-D wavelet transform. Given that there are some sample texture images from the same kind of texture, these images should have the same spatial relation between neighborhood pixels as this texture. These images are all decomposed to obtain the same frequency regions by 2-D wavelet transform.

The frequency channel is defined as each branch of the tree. For all frequency channels, the energy values are stored at the leaf and make these energy values to compose a vector of length ' $k$ ', called the channel energy vector ' $\mathrm{V}$ ', where ' $\mathrm{k}$ ' is frequency channels of image. The value of $\mathrm{k}$ can be calculated as,

$\mathrm{K}=4^{\mathrm{n}}, \mathrm{n} \rightarrow$ Number of decomposition levels

In a single texture, samples are acquired by taking overlap of 32 pixels vertically and horizontally. The channel energy matrix is formed by using ' $j$ ' number of channel energy vectors of length ' $k$ '. These channel energy vectors are obtained by decomposing the ' $\mathrm{j}$ ' number of samples using 2-D wavelet packet transform. The channel energy matrix is represented as $\mathrm{M}_{\mathrm{jxk}}$, where ' $\mathrm{j}$ ' is number of rows and ' $\mathrm{k}$ ' is number of columns.

The covariance matrix $\mathrm{C}_{\mathrm{k} \times \mathrm{k}}$ is obtained from the channel energy matrix $M_{j x k}$, where $C_{i j}$ is the correlation coefficient between the $\mathrm{i}^{\text {th }}$ and $\mathrm{j}^{\text {th }}$ channel frequency. The correlation is used for finding the similarities between two images. This assumption can be tested by the following experiment.

We acquire 81 sample images of size $128 \mathrm{x}$ 128 with an overlap of 32 pixels between vertically and horizontally adjacent images of size $640 \times 640$ with 256 gray levels, respectively. They are very homogeneous. Next, two 64 × 64 covariance matrices can be obtained. As descends, the top ten channel pairs can be extracted. It can be observed that the correlation between different frequency channels certainly exists. This experiment implies that the correlation between frequency channels is a distinctive characteristic of the texture and can serve as a good candidate for texture representation to detect different texture. The preprocessing steps is needed to output distinctive top channel pairs of a texture for texture classification. The steps is given as follows.

\section{Preprocessing Phase}

[Input:] all samples of a given texture

[Output:] the channel-pair list and the channelenergy matrix

All the ' $\mathrm{j}$ ' number of samples of a texture is given as the input. These samples are decomposed by using two dimensional wavelet packet transform till the size of sub image is $16 \times 16$. This decomposition process gives ' $\mathrm{k}$ ' number of frequency channels. For these frequency channels, the energy values are calculated and these values are used for forming the channel energy vector ' $\mathrm{V}$ ' of 
length ' $k$ '. The ' $j$ ' number of channel energy vectors of the ' $\mathrm{j}$ ' samples from a single texture are used to construct a channel energy matrix of size $\mathrm{j} \mathrm{x} \mathrm{k}$. If the correlation coefficient is greater than or equal to the threshold value, then that channel pairs are selected and these channel pairs are arranged in descending order. From these channel pairs the top ten channel pairs are selected. The channel pair list and the channel energy matrix are the output of this process.

\section{Linear Regression Model}

In the above subsection, the correlation between different frequency regions has been validated as a sort of effective texture characteristic. In this subsection, we employ the simple linear regression model to analyze the correlation. If a set of random data $\left(\mathrm{x}_{1}\right.$, $\left.\mathrm{y}_{1}\right)^{\mathrm{T}}, \quad\left(\mathrm{x}_{2}, \mathrm{y}_{2}\right)^{\mathrm{T}}, \ldots \ldots \ldots . .\left(\mathrm{x}_{\mathrm{n}}, \mathrm{y}_{\mathrm{n}}\right)^{\mathrm{T}}$ for two numerical variables $\mathrm{X}$ and $\mathrm{Y}$, where $\mathrm{X}$ is a cause of $\mathrm{Y}$. In this linear regression analysis, the distribution of the random data appears as a straight line in $\mathrm{X}, \mathrm{Y}$ space when $\mathrm{X}$ and $\mathrm{Y}$ are perfectly related linearly. This captures a relationship between two variables. This line function can be given as,

$$
\hat{y}=a x+b
$$

Here, the linear regression model is used to extract the texture features from the correlation in the frequency channel pairs. The energy values of two frequency channels of one of the channel pair in the top ten list are taken from the channel energy matrix $M$ and consider these energy values as the random data $\left(x_{1}, y 1\right)^{\mathrm{T}}$, $\left(\mathrm{x}_{2}, \mathrm{y}_{2}\right)^{\mathrm{T}}, \ldots \ldots \ldots . .\left(\mathrm{x}_{\mathrm{n}}, \mathrm{y}_{\mathrm{n}}\right)^{\mathrm{T}}$ for two variables $\mathrm{X}$ and $\mathrm{Y}$ represent a straight line in $\mathrm{X}, \mathrm{Y}$ space.

The parameters ' $a$ ' and ' $b$ ' of the line can be calculated using the expressions,

$$
\begin{aligned}
\mathrm{a}= & \mathrm{n} \sum_{\mathrm{i}=1}^{n} x i y^{x}-\sum_{\mathrm{i}=1}^{n} x i \sum_{\mathrm{i}=1}^{n} y i / \mathrm{n} \\
& \sum_{i=1}^{n} x i^{2}-\left(\sum_{i=1}^{n} x i\right)^{2} \\
\mathrm{~b}= & 1 / \mathrm{n} \sum_{i=1}^{n} y i-\mathrm{a} / \mathrm{n} \sum_{i=1}^{n} x i
\end{aligned}
$$

When high correlation data is taken into the regression to obtain $\hat{y}$, there exists the residual between $\mathrm{y}$ and $\hat{y}$, such residuals exhibit a normal distribution curve. The normal distribution parameters are mean $(\mu)$ and standard deviation $(\sigma)$. The values of $\mu$ and $\sigma$ can be given as,

$$
\begin{aligned}
& \mu=\sum_{i=1}^{n}\left(y i-y i^{\wedge}\right) / \mathrm{n} \\
& \sigma=\sqrt{\sum_{i=1}^{n}\left(y i-y i^{\wedge}\right)^{2} / n-2}
\end{aligned}
$$

PSWT loses the middle and high frequency information. TSWT does not take into account the correlation between different frequency channels. In contrast, our method not only thinks about all frequency channels but also analyzes the correlation between them with the simple linear regression model. So, it can be viewed as a very useful multiresolution method.

\section{TEXTURE CLASSIFICATION A. Learning Phase}

[Input:] all samples of all textures [Output:] the feature lists of all textures

Given all samples obtained from the same texture, get the channel-pair list and the channel-energy matrix of this texture by the preprocessing algorithm.

With the channel-pair list and the channelenergy matrix $\mathrm{M}$, figure out the parameters a, $b, \mu$, and $\sigma$ of each top channel pair by using the expressions. Consider the parameters $\mathrm{a}, \mathrm{b}$, $\mu$ and $\sigma$, two frequency channels and the correlation coefficient $\rho$ of each top channel pair and the index of this texture as a feature of this texture. Put all such features into a list as $\rho$ descends, called the feature list, and then insert this list into the database. 
Note that the feature lists of the textures are needed to store into the database in the third step. In the feature lists, every texture feature contains the parameters $a, b, \mu$, and $\sigma$, two frequency channels and the correlation coefficient $\rho$ of one channel pair and the index of a texture. The parameters $a, b$ and two frequency channels are used to compute the residual of an unknown texture image at a top channel pair of a texture, and the parameters $\mu$ and $\sigma$ are used to get the threshold $\mu \pm 3 \sigma$ in order to determine whether this image satisfies the correlation at this top channel pair of this texture as discussed in Section III.

\section{B. Classification Phase}

[Input:] an unknown texture image and the feature lists in the database

[Output:] the index of texture to which this unknown texture image is assigned

An unknown texture image is decomposed into number of sub images and the features of each sub images are extracted. This feature list and unknown texture are given as input. These features are compared with the top 10 channel pairs feature list in the database and then the two identical frequency channels are selected. Take one energy of two channels as xi into the linear regression equation (line function) and the residual value is calculated by $\mid \mathrm{yi}^{\wedge}{ }^{\wedge}$-yi|, where yi is the other energy. If the residual value is in between $\mu+3 \sigma$ and $\mu-3 \sigma$, then the corresponding texture is considered. If the residual value is beyond $\mu \pm 3 \sigma$, then that texture is neglected.

\section{EXPERIMENTAL RESULTS}

In this section, we will verify the performance of the classification algorithm discussed in Section III. We used 3 textures, as shown in Fig., obtained from the Brodatz's texture album. Every original image is of size $640 \mathrm{x}$ 640 pixels with 256 gray levels. 81 sample images of size 128 x 128 with an overlap of 32 pixels between vertically and horizontally adjacent images are extracted from each original image and used in the experiments. These 81 texture images are separated to two sets being used as the training set with 41 images and the test set with 40 images, respectively. Three set of classes are obtained from the Brodatz's texture album.

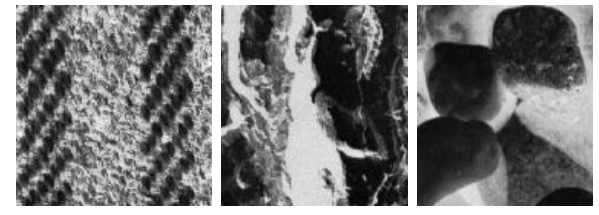

Fig.3.Sample Textures
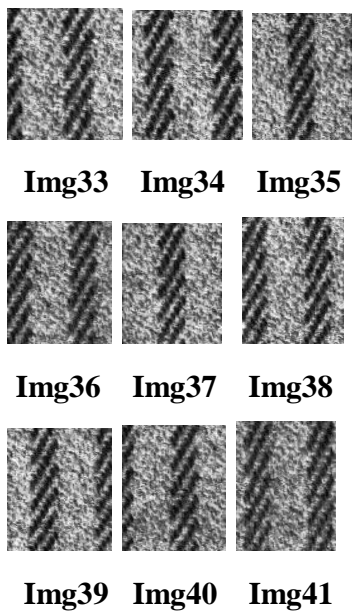

4.(a)

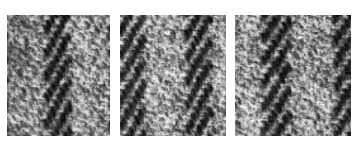

Img32 Img33 Img34

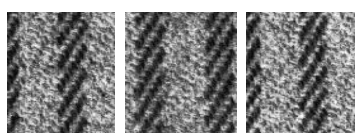

Img35 Img36 Img37 


\section{Img38 Img39 Img40}

4.(b)

Fig.4.(a)Subsamples for training

(b)Subsamples for testing

\begin{tabular}{|l|c|c|c|}
\hline & $\begin{array}{c}\text { Linear } \\
\text { Wavelet }\end{array}$ & $\begin{array}{c}\text { Four Dierctional } \\
\text { Lifting }\end{array}$ & $\begin{array}{c}\text { Eight Directional } \\
\text { Lifiting }\end{array}$ \\
\hline Classification Rate & $50 \%$ & $60 \%$ & $61 \%$ \\
\hline
\end{tabular}

Table.1.Classificaction Rates

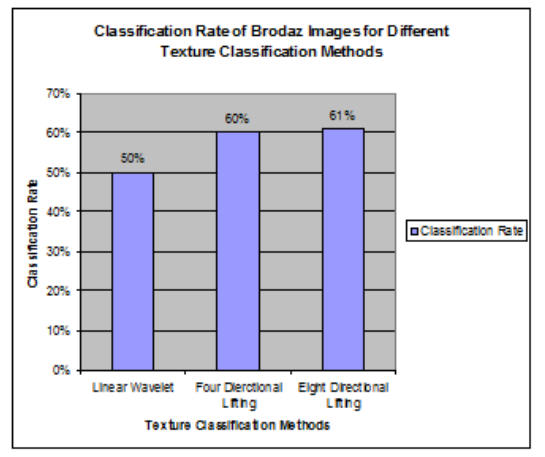

Fig 6.Comparison chart

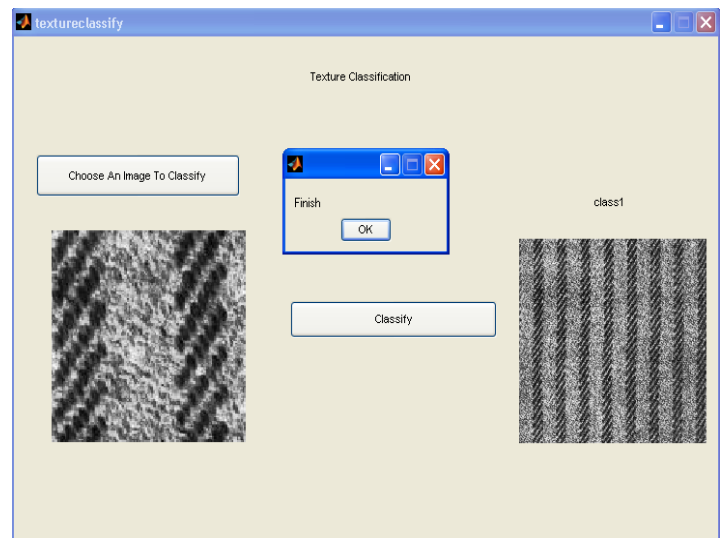

Fig 7.Texture Classification Output 
Table.2.Features Stored in the database

\begin{tabular}{|c|c|c|c|c|c|c|}
\hline \multicolumn{7}{|c|}{ learnfeatures } \\
\hline class & $\begin{array}{c}\text { xchannelna } \\
\text { me }\end{array}$ & $\begin{array}{c}\text { ychannelna } \\
\text { me }\end{array}$ & afea & bfea & mean & std \\
\hline class 1 & ODBC & OBDC & $\begin{array}{r}1.076999890486 \\
35\end{array}$ & 7.45780900818872 & $\begin{array}{r}0.69468466070 \\
0578 \\
\end{array}$ & 0.834577417614148 \\
\hline class1 & ODBA & ODAA & $\begin{array}{r}0.593474246769 \\
77\end{array}$ & 0.423906676764114 & \begin{tabular}{|r|}
0.43687069656 \\
5506 \\
\end{tabular} & 0.540738248722123 \\
\hline class 1 & ODBB & ODBA & 1.039348196656 & $-1.41450979287328 \mathrm{E}-03$ & $\begin{array}{r}0.69408562741 \\
9633\end{array}$ & 0.830444908388491 \\
\hline class1 & ODBB & ODAA & $\begin{array}{r}0.667394736341 \\
754\end{array}$ & -0.155054138339636 & $\begin{array}{r}0.46246866026 \\
677\end{array}$ & 0.589836046068266 \\
\hline class1 & ODCD & ODAA & $\begin{array}{r}0.669814131418 \\
217\end{array}$ & -1.19222121741298 & $\begin{array}{r}0.47424467535 \\
3011 \\
\end{array}$ & 0.594036321064595 \\
\hline class1 & ODCA & ODAA & $\begin{array}{r}0.823662186029 \\
584\end{array}$ & -0.219467726347863 & \begin{tabular}{|r|}
0.46478898767 \\
7635 \\
\end{tabular} & 0.594224585903574 \\
\hline class1 & ODDD & ODBB & $\begin{array}{r}0.862325170210 \\
047\end{array}$ & -1.30871695970843 & $\begin{array}{r}0.65181598572 \\
3344 \\
\end{array}$ & 0.797325813899116 \\
\hline class1 & ODAB & ODAA & $\begin{array}{r}0.838288138050 \\
009\end{array}$ & -0.246919933605523 & $\begin{array}{r}0.49079208699 \\
9362 \\
\end{array}$ & 0.60598780269064 \\
\hline class1 & ODCD & ODAC & $\begin{array}{r}0.667976805117 \\
044\end{array}$ & -0.199053811391728 & \begin{tabular}{|r|}
0.49707567387 \\
9373
\end{tabular} & 0.622000758709531 \\
\hline class1 & ODBD & OCBA & $\begin{array}{r}0.810874644327 \\
303\end{array}$ & 5.11709945879494 & $\begin{array}{r}0.60957040975 \\
8177\end{array}$ & 0.755816144268875 \\
\hline
\end{tabular}

\section{CONCLUSION}

In texture analysis and classification with the simple linear regression model based on the directional lifting based wavelet transform, the classification rate is improved. In the multiresolution analysis the energy values are computed directly from the subimages and extract the features to characterize the texture image at the multidimension space and yet this method employs the correlation between different frequency regions to construct the texture feature so it employs the threshold comparison rather than some distance measurement methods. Therefore it is very easy and fast to examine the change of different frequency channels for texture image. Classification rate is improved. Correlation is considered, so accuracy is high. 


\section{REFERENCES}

[1]Baocai Yin,Xin Li,Yunhui Shi,Feizhou Zhang,Nan Zhang,'Directional lifting-based wavelet transform for multiple description image coding", signalprocessing: Imagecommunication 23(2008) 42-57.

[2] J. Zhang and T. Tan, "Brief review of invariant texture analysis Methods", Pattern Recognition., vol. 35, pp. 735747, 2002.

[3] Y. Rui, T. S. Huang, and S. F. Chang, "Image retrieval: Current techniques, promising directions, and open issues," J. Vis. Commun. Image Represent., vol. 10, pp. 39-62, 1999.

[4] R. Randen and J. H. Husøy, "Filtering for texture classification:A comparative study," IEEE Trans. Pattern Anal. Mach. Intell., vol. 21, no. 4,pp. 291-310, Apr. 1999.

[5] G. H. Wu, Y. J. Zhang, and X. G. Lin, "Wavelet transform-based texture classification with feature weighting," Proc. IEEE Int. Conf. Image Processing, vol. 4, no. 10 , pp. 435-439, Oct. 1999.

[6] C.-C. Chen and C.-C. Chen, "Filtering methods for texture discrimination," Pattern Recognition, vol. 20, pp. 783-790, 1999.

[7] W. Y. Ma and B. S. Manjunath, "Texture features and learning similarity," Proc. IEEE CVPR, no. 18-20, pp. 425-430, Jun. 1996.

[8]M. Unser, "Texture classification and segmentation using wavelet frames," IEEE Trans. Image Process., vol. 4, no. 11, pp. 1549-1560, Nov. 1995.
[9] W. Y. Ma and B. S. Manjunath, "A comparison of wavelet transform features for texture image annotation," Proc. IEEE Int. Conf. Image Processing, vol. 2, no. 23-26, pp. 256-259, Oct. 1995.

[10] T. Chang and C.-C. J. Kuo, "Texture analysis and classification with tree-structured wavelet transform," IEEE Trans. Image Process., vol.2, no. 4, pp. 429-441, Oct. 1993.

[11] T. Chang and C.-C. J. Kuo, "Tree-structured wavelet transform for textured image segmentation," Proc. SPIE, vol. 1770, pp. 394-405, 1992.

[12] T. Chang and C.-C. J. Kuo, "A wavelet transform approach to texture analysis," in Proc. IEEE ICASSP, Mar. 1992, vol. 4, no. 23-26, pp.661-664.

[13] M. Unser, "Local linear transforms for texture measurements," Signal Process., vol. 11, pp. 61-79, 1986.

[14] P. C. Chen and T. Pavlidis, "Segmentation by texture using correlation,"IEEE Trans. Pattern Anal. Mach. Intell., vol. 5, no. 1, pp. 64-69,Jan. 1983.

[15] R. L. Kashyap and R. Chellappa, "Estimation and choice of neighbors in spatial-interaction models of images," IEEE Trans. Inf. Theory, vol.29, no. 1, pp. 60-72, Jan. 1983.

[16] R. M. Haralick, K. Shanmugan, and I. Dinstein, "Textural features for image classification," IEEE Trans. Syst. Man Cybern., vol. SMC-6, no.6, pp. 610-621, Nov. 1973. 